\section{Computer technology update}

COMPUTER n. Automatic electronic apparatus for making calculations or controlling operations that are expressible in numerical or logical terms; hence IZE v.t. equip with, perform by, produce by, computer.

\section{Why digital?}

Imagine, for a moment, that you need to send a message to me; you need to tell me whether to come and rescue you, stay where I am, or send for reinforcements. Your life, and those of others, depend on correct transmission of this message which must be sent using a flashlight and one of two codes. The first code associates each option with a particular number of flashes of the torch - one for rescue, two for stay and three for reinforcements. The second code depends on the use of a slider at the side of the torch to vary the strength of the beam; the three possible messages are respectively represented by a weak signal, a medium-strength one, and a bright one. Which coding system would you choose? I'd go for the number-of-flashes option; there's little chance of misinterpretation (provided both you and I understand the code and can count). The other, intensity-of-beam, method leaves plenty of room for confusion: your "bright" may be my "medium" and our attempts to communicate may end up like those of Allan Donald and Lance Klusener. A coding system that, when broken down into its most basic sub-units, depends on a signal that is either on or off and pays no attention to the strength of the signal, is a digital one. It is their digital nature that has made modern computers so reliable and successful. Why then, you may well ask, does your computer "freeze" so often? Remember, the reliability of the number-of-flashes method of communicating by torch is negated if the sender and receiver are badly trained and don't understand the code properly or can't count. Similarly, badly written software can "crash" even a reliable digital computer.

\section{What's what}

Let's take a look at the basic components of a computer. The most important part of the computer is the least impressive-looking: the "box", usually an unchallenging beige colour (but this is changing as computers become fashion accessories). It has many wires applied to its back, one or more slits and one or more lights on its front, and makes whirring or clicking noises at times. Within this box live several components which, being important, will now be introduced.

\section{The CPU (Central Process- ing Unit)}

The brain of the computer. Takes an incoming stream of 0 's and l's (data) and, according to instructions (also encoded as a series of 0 's and l's) given to it by a program, manipulates this data and sends out a stream of ... yes: 0 's andl's. For example if two "pieces" of data, 001 (the number one) and 010 (two), were to arrive at some fictitious processor with the instruction 


\section{TECHNOLOGY UPDATE}

\section{from page 25}

to add them, the processor would spit out 011 (three). If the data arriving at the processor were a very, very long string of 0 's and l's representing a digitised picture of your child, and a series of instructions went to the processor telling it to increase the contrast in that picture, the ultimate result would be another very, very long string of 0's and l's representing a more contrasty picture of your child.

\section{Memory (RAM - Random Access Memory - and Hard Drive)}

Picture a craftsman in his workshop. Around his waist is a leather pouch in which he holds those tools that he is currently using. Those tools that he is not actively using are stored on shelves that line one of the walls of his workshop. The leather pouch is similar to the RAM of a computer, the short-term memory that holds those parts of programs and scraps of data that are currently needed by the processor. This memory communicates with the processor very quickly, but has far less capacity than the hard drive and is "volatile" - when power supply to the computer is lost, all data in RAM is lost. The hard drive is similar to the shelving in our craftsman's workshop. It has a large capacity (perhaps a hundred times that of RAM) but fetching data from the hard drive takes much longer than fetching it from RAM, just as getting a screwdriver from the shelf takes longer than simply pulling it out of the pouch. Data stored on a hard drive remains there when the computer is switched off.

\section{Motherboard}

If, feeling bored or adventurous, you decide to open your computer and take a peep inside you will see, among other things, a number of cards or boards. The exact number will depend on what you have added to your system, but one that will definitely be there is the "motherboard". This holds the "guts" of the computer - including the CPU and RAM - and may also hold the video (things that appear on your monitor) and sound controllers, which are then said to be "on-board".

\section{Video card}

In the bad old days of DOS your computer's screen may, at a particular time, have been completely blank apart from some witty and enticing statement such as: $\mathrm{C}:>$ Taken by the eloquence of this you might have been tempted to type: dir. This would have resulted in a whole lot of print appearing on your screen - and that's about as complex as things got, video-wise, in those days. Onscreen graphics were confined to thrilling stuff like arranging x's, o's and (for the bold) -'s into patterns to depict Mickey Mouse. Nowadays the screen is decorated, even in its "resting" state with thousands (or millions) of colours, littered with icons which await the click of your mouse to launch programs that allow you to show your holiday photos or videos, or (I suppose it must be said, this being a Radiology journal) the chest $\mathrm{x}$-ray of a patient who is a hundred kilometres from your rooms. All this involves a huge amount of processing, a task which is shunted away from the main processor to a separate video module, with its own RAM, and often to be found on a separate card plugged into the motherboard.

\section{Sound card}

The sound producing capabilities of home computers have increased from cryptic beeping to the ability to play the overture from Tannhauser (why do we get such a kick out of listening to music over the tinny little speakers that come with our computers? Beats me, but I do it too). As with video, this intense task is often handled by a separate card.

\section{Modem}

If you want to be able to say "yes" when asked "Do you have the internet?" you need a modem, your portal to all the information, sleaze and viruses out there. The modem converts data (including pictures and sound) between the digital type that a computer understands and the analogue type that the phone lines demand.

\section{Keyboard, monitor, mouse}

The purpose of these is self-evident and, for the moment, we'll ignore them.

\section{What shall I buy?}

I will not try to give specific advice regarding the features to be desired in a computer. Such an attempt would be futile, firstly because needs differ from person to person and secondly because the pace of progress would guarantee the obsolescence of the recommendations by the time of their publication. But, some may find a few general pointers helpful.

\section{Processor}

Names like "Pentium" and "PIII" each denote a class of processors whose members have similar characteristics and capabilities but operate at different "clock speeds". The clock speed of the processor is indicated by a number like 400 $\mathrm{MHz}$. The performance advantage of a processor which is faster but is within the same class as another (e.g. a PIII $500 \mathrm{MHz}$ versus a PIII $450 \mathrm{MHz}$ ) is fairly predictable and is roughly proportional to the size of the number. The performance advantage of a more recent class of processor (e.g. a PIII versus a PII) is less easy to predict and is more dependent on what you do with your computer - but it is usually considerable. My general advice when buying 


\section{TECHNOLOGY UPDATE}

\section{from page 26}

a new desktop computer would be to buy into the latest class of processor but to look for the "sweet spot" (the point at which diminishing returns for your money start to set in) with regard to processor speed. It is really not going to make much difference, except for the most demanding of applications, whether your processor is a $550 \mathrm{MHz}$ or a 500 , and there's usually a considerable premium to be paid for being at the cutting-edge.

\section{RAM}

Let's refer back to our analogy above, which equates RAM with a leather tool-pouch and hard drives with shelving. Imagine a very slick craftsman - he's a very fast worker but has only a small pouch to hold tools. He has to stop what he's doing repeatedly to fetch things from the shelves. He'll probably take longer to get a job done than would a slower worker with a larger tool-pouch. Similarly, it's no good having the fastest processor available but being short on RAM. In my opinion, if it's worth having a modern processor, like a PIII (or its non-Intel equivalents), then it's worth having at least $64 \mathrm{MB}$ of RAM.

\section{Hard drive}

It used to be as inevitable as death and taxes that you would run out of disk space within a year or two of buying a computer. This remains largely true if you are using your computer to do all the things that a modern computer can do, for example to manipulate and store pictures from a scanner or digital camera. If all you're doing is a little word processing and using the occasional spreadsheet you'll have a tough time trying to fill one of the modern multi-gigabyte-size hard drives. But if this is all you're doing, you don't really need a modern "powerhouse" computer. Where then, you may ask, can you buy a little, cheap computer with specifications and software honed down to your limited needs? You can't. The industry says you have to do more with your computer. Get cracking. 